\title{
Gerholz, Karl-Heinz
}

\section{Potenziale von Service Learning in den Wirtschaftswissenschaften - Didaktische Konzeption und empirische Befunde aus einer Ex-ante-Analyse}

Seifried, Jürgen [Hrsg.]; Seeber, Susan [Hrsg.]; Ziegler, Birgit [Hrsg.]: Jahrbuch der berufs- und wirtschaftspädagogischen Forschung 2015. Opladen ; Berlin ; Toronto : Verlag Barbara Budrich 2015, S. 117-130. (Schriftenreihe der Sektion Berufs- und Wirtschaftspädagogik der Deutschen Gesellschaft für Erziehungswissenschaft (DGfE))

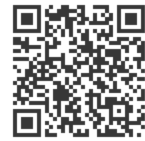

Quellenangabe/ Reference:

Gerholz, Karl-Heinz: Potenziale von Service Learning in den Wirtschaftswissenschaften - Didaktische Konzeption und empirische Befunde aus einer Ex-ante-Analyse - In: Seifried, Jürgen [Hrsg.]; Seeber, Susan [Hrsg.]; Ziegler, Birgit [Hrsg.]: Jahrbuch der berufs- und wirtschaftspädagogischen Forschung 2015. Opladen ; Berlin ; Toronto : Verlag Barbara Budrich 2015, S. 117-130 - URN:

urn:nbn:de:0111-pedocs-183484 - DOI: 10.25656/01:18348

https://nbn-resolving.org/urn:nbn:de:0111-pedocs-183484

https://doi.org/10.25656/01:18348

in Kooperation mit / in cooperation with:

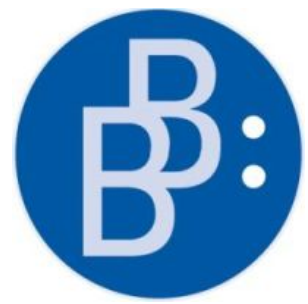

https://www.budrich.de

\section{Nutzungsbedingungen}

Gewährt wird ein nicht exklusives, nicht übertragbares, persönliches und beschränktes Recht auf Nutzung dieses Dokuments. Dieses Dokument ist ausschließlich für den persönlichen, nicht-kommerziellen Gebrauch bestimmt. Die Nutzung stellt keine Übertragung des Eigentumsrechts an diesem Dokument dar und gilt vorbehaltlich der folgenden Einschränkungen: Auf sämtlichen Kopien dieses Dokuments müssen alle Urheberrechtshinweise und sonstigen Hinweise auf gesetzlichen Schutz beibehalten werden. Sie dürfen dieses Dokument nicht in irgendeiner Weise abändern, noch dürfen Sie dieses Dokument für öffentliche oder kommerzielle Zwecke vervielfältigen, öffentlich ausstellen, aufführen, vertreiben oder anderweitig nutzen.

Mit der Verwendung dieses Dokuments erkennen Sie die Nutzungsbedingungen an.

\section{Terms of use}

We grant a non-exclusive, non-transferable, individual and limited right to using this document.

This document is solely intended for your personal, non-commercial use. Use of this document does not include any transfer of property rights and it is conditional to the following limitations: All of the copies of this documents must retain all copyright information and other information regarding legal protection. You are not allowed to alter this document in any way, to copy it for public or commercial purposes, to exhibit the document in public, to perform, distribute or otherwise use the document in public.

By using this particular document, you accept the above-stated conditions of use.

\section{Kontakt / Contact:}

peDOcs

DIPF | Leibniz-Institut für Bildungsforschung und Bildungsinformation

Informationszentrum (IZ) Bildung

E-Mail: pedocs@dipf.de

Internet: www.pedocs.de

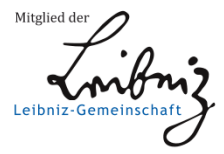




\section{Jahrbuch der berufs- und wirtschaftspädagogischen Forschung 2015}

Jürgen Seifried, Susan Seeber, Birgit Ziegler (Hrsg.)

DGfE Deutsche Gesellschatt 
Schriftenreihe der Sektion

Berufs- und Wirtschaftspädagogik

der Deutschen Gesellschaft

für Erziehungswissenschaft (DGfE) 
Jürgen Seifried

Susan Seeber

Birgit Ziegler (Hrsg.)

Jahrbuch der berufs- und

wirtschaftspädagogischen

Forschung 2015

Verlag Barbara Budrich

Opladen • Berlin • Toronto 2015 
Bibliografische Information der Deutschen Nationalbibliothek

Die Deutsche Nationalbibliothek verzeichnet diese Publikation in der Deutschen

Nationalbibliografie; detaillierte bibliografische Daten sind im Internet über

http://dnb.d-nb.de abrufbar.

Gedruckt auf säurefreiem und alterungsbeständigem Papier.

Alle Rechte vorbehalten.

C 2015 Verlag Barbara Budrich, Opladen, Berlin \& Toronto

www.budrich-verlag.de

$\begin{array}{ll}\text { ISBN } & 978-3-8474-0722-5 \text { (Paperback) } \\ \text { eISBN } & 978-3-8474-0871-0 \text { (eBook) }\end{array}$

Das Werk einschließlich aller seiner Teile ist urheberrechtlich geschützt. Jede Verwertung außerhalb der engen Grenzen des Urheberrechtsgesetzes ist ohne Zustimmung des Verlages unzulässig und strafbar. Das gilt insbesondere für Vervielfältigungen, Übersetzungen, Mikroverfilmungen und die Einspeicherung und Verarbeitung in elektronischen Systemen.

Umschlaggestaltung: Bettina Lehfeldt, Kleinmachnow - www.lehfeldtgraphic.de Typographisches Lektorat: Anja Borkam, Jena 


\section{Inhaltsverzeichnis}

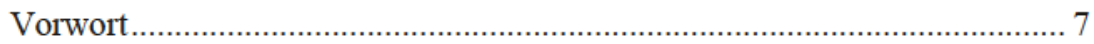

\section{Teil I: Kompetenz(facetten) und Persönlichkeitsentwicklung}

Carmela Aprea, Eveline Wuttke, Seraina Leumann, Michael Heumann Kompetenzfacetten von Financial Literacy: Sichtweisen verschiedener Akteure

Bärbel Fürstenau, Mandy Hommel, Claudia Leopold, Héctor Ponce, Mario López

Baufinanzierung nach Maß? - Aufbau von Finanzkompetenz durch

Online-Informationen 23

Sebastian Lerch

Das kompetente Selbst. Empirische Befunde zu Selbstkompetenzen in Ingenieurwesen, Pädagogik und Medizin

Raphaela Schreiber

Berufliche Identität von Zeitarbeitnehmern

\section{Teil II: Forschung zu Übergängen}

Sylvia Rahn, Thorsten Bührmann, Emanuel Hartkopf

Geplantes Verhalten im Übergangsprozess? - Berufsorientierungs- und Übergangsprozesse von Schülerinnen und Schülern einjähriger Bildungsgänge des Übergangssegments

Taiga Brahm

Resilienzförderung im Übergangssegment - Erste Ergebnisse einer Längsschnittuntersuchung.

Bernd Fitzenberger, Stefanie Licklederer, Markus Zimmermann Übergänge von der allgemeinbildenden Schule in berufliche Ausbildung und Arbeitsmarkt: Die ökonomische Perspektive 


\section{Teil III: Unterrichts-, Hochschul- und Lehrerbildungsforschung}

Nicole Kimmelmann, Katja Dippold-Schenk

Professionalisierung von Lehrpersonen in der beruflichen

Weiterbildung als didaktische Herausforderung - Erste Ergebnisse aus dem Verbundprojekt SpraSibeQ

Karl-Heinz Gerholz

Potenziale von Service Learning in den Wirtschaftswissenschaften Didaktische Konzeption und empirische Befunde aus einer Ex-anteAnalyse

Matthias Conrad, Stephan Schumann

Tablet-PCs im Wirtschaftsunterricht und die Rolle der Lehrperson

\section{Teil IV: Historische Berufsbildungsforschung}

Dieter Hölterhoff, Peter Kuklinski

Vergleichende Untersuchung der ersten Berufsschulverordnungen der 1990er Jahre in den ostdeutschen Ländern - Gemeinsamkeiten und Unterschiede

Herausgeberschaft

Autorinnen und Autoren 


\section{Potenziale von Service Learning in den Wirtschaftswissenschaften - Didaktische Konzeption und empirische Befunde aus einer Ex-ante-Analyse}

Karl-Heinz Gerholz

\section{Hinführung}

Service Learning ist eine Lernform, die fachwissenschaftliche Inhalte des Studiums mit gemeinnützigem Engagement verbindet. In der internationalen wie auch in der deutschsprachigen Universitätslandschaft hält Service Learning in den letzten Jahren vermehrt Einzug (vgl. u. a. Backhaus-Maul \& Roth 2013). Damit werden die Potenziale verbunden, nicht nur einen Beitrag für die fachlich-methodische Kompetenzentwicklung der Studierenden zu leisten, sondern darüber hinaus die Persönlichkeitsentwicklung im Studium durch das Element des gemeinnützigen Engagements in den Blick zu nehmen.

Wenngleich die Potenziale von Service Learning sich in empirischen Untersuchungen aus dem US-amerikanischen Raum widerspiegeln (vgl. dazu Abschnitt 2.2), ist einerseits näher zu elaborieren, inwiefern dieses auch für den deutschsprachigen Kontext evident ist. Bildungsinstitutionen im USamerikanischen Raum weisen traditionell eine stärkere Gemeinwohl-Orientierung auf. Universitäten werden als öffentliche Institutionen interpretiert, die den Interessen der Bürger folgen (vgl. u. a. Muller 1999). Andererseits ist ein Forschungsdesiderat hinsichtlich des Zusammenhangs der didaktischen Gestaltung von Service Learning-Arrangements und deren Wirkung festzuhalten. In der Regel werden in den vorliegenden Studien die Lernergebnisse der Studierenden als Bezugspunkt genommen, aber weniger die Zusammenhänge zur fachdidaktischen Ausgestaltung des Service Learnings aufgegriffen. Vor allem für wirtschaftswissenschaftliche Studiengänge - und Bildungsgänge - können hierüber Hinweise für die Gestaltung von Service Learning-Arrangements generiert werden.

Zielstellung des vorliegenden Aufsatzes ist es, zunächst die didaktische Grundkonzeption von Service Learning vorzustellen und die dazu vorliegenden empirischen Ergebnisse zu analysieren (Abschnitt 2). Darauf basierend werden empirische Befunde aus einer Pilotstudie zu einem Service LearningModul in der wirtschaftswissenschaftlichen Hochschulbildung hinsichtlich der Gründe für die Modulwahl und die Wahrnehmung der didaktischen Kohärenz seitens der Studierenden vorgestellt (Abschnitt 3). 


\section{Service Learning: Didaktische Konzeption und Wirksamkeit}

\subsection{Service Learning als Kontur einer Methode}

Beim Service Learning wird der Lernprozess der Studierenden über die Bearbeitung von gemeinnützigen Problemstellungen arrangiert, was einen Service für die Kommune darstellt. Über die Reflexion der Erfahrungen soll ein elaboriertes Verständnis der Studieninhalte gefördert sowie eine Sensibilisierung für gesellschaftliches Engagement vorgenommen werden. So definieren Bringle \& Clayton (2012, S. 105) Service Learning als "course or competency-based, credit-bearing educational experience in which students (a) participate in mutually identified service activities that benefit the community, and (b) reflect on the service activity in such a way as to gain further understanding of course content, a broader appreciation of the discipline, and an enhanced sense of personal values and civic responsibility." Neben fachlichen, sozial-kommunikativen und methodischen Fähigkeiten soll ein Beitrag zur Persönlichkeitsentwicklung der Studierenden geleistet werden. Darüber hinaus wird die curriculare Integration von Service Learning betont.

Die Lernform Service Learning kann als eine didaktische Kontur verstanden werden, worunter handlungsorientierte, problembasierte oder forschungsorientierte Lernformen subsumiert werden können. Es handelt sich dabei nicht nur um ein didaktisches Konzept für die Hochschulbildung, sondern bietet auch Möglichkeiten auf Ebene der allgemeinbildenden und beruflichen Schulen. Die Besonderheit liegt in der Verknüpfung des Lernprozesses mit einem realen Bedürfnis aus der Kommune. Leitend für den Gedanken der Förderung des kommunalen Engagements sind $u$. a. die Arbeiten von Dewey. Für Dewey ist die Sensibilisierung der Lernenden für ihre Rolle als verantwortliche Bürger in einer demokratischen Gemeinschaft ein Ziel von Bildungsprozessen. Eine Schlussfolgerung ist für ihn, die Lernprozesse in die kommunalen Bedürfnisse und die damit verbundenen Problemstellungen einzubetten (vgl. Dewey 1966).

Die Arbeiten von Dewey und den Diskurs um Service Learning im USamerikanischen Raum führen Godfrey, Illes und Berry (2005) zu drei Elementen zusammen, die für Service Learning konstituierend sind:

1. Realität (Reality): Die zu bearbeitende Problemstellung der Studierenden sollte nicht nur an den realen Bedürfnissen der Kommune andocken, sondern die Studierenden sollten mit möglichst unterschiedlichen sozialen Herausforderungen (wie z. B. Armut, Obdachlosigkeit) konfrontiert werden, um die Widersprüchlichkeiten und Vieldimensionalität sozialer Belange zu erfahren. Die Verknüpfung von Studieninhalten mit realen sozialen Fragestellungen ermöglicht es den Studierenden, an der Verbes- 
serung der Gesellschaft mitzuwirken und ihr Handeln als wirksam zu erleben.

2. Gegenseitigkeit (Reciprocity): Service Learning soll eine partnerschaftliche Lernerfahrung sein, indem die Studierenden kooperativ mit den kommunalen Partnern an sozialen Herausforderungen arbeiten. Die Annahme ist dabei, dass die kommunalen Partner wie die Studierenden über ein unterschiedliches konzeptionelles Wissen und Erfahrungswissen verfügen und die gegenseitige Bezugnahme einen Mehrwert bei der Lösung der sozialen Problemstellung erzielt.

3. Reflexion (Reflection): Die Wirkung des Service Learnings auf die Kompetenzentwicklung der Studierenden hängt in hohem Maße von der Reflexion der Erfahrungen ab. Die Studierenden sollen angeregt werden, nicht nur die Verbindungen zwischen ihren Service-Erfahrungen und den Inhalten des Studiums herzustellen, sondern auch ein eigenes Verständnis und ihre persönlichen Ansichten für soziale Herausforderungen entwickeln. Die Reflexion ermöglicht die Verknüpfung der äußeren Service-Herausforderung und der inneren Einstellungen der Studierenden. Dewey spricht hierbei von der ,organic connection' zwischen ServiceErfahrung und Persönlichkeitsentwicklung (vgl. Dewey 1998).

Die drei Elemente Realität, Gegenseitigkeit und Reflexion können als Orientierungsschablone für die didaktische Gestaltung von Service Learning-Arrangements fungieren. Hierbei wird deutlich, dass beim Service Learning zwischen dem Serviceprozess und dem Lernprozess zu differenzieren ist (vgl. Gerholz \& Losch 2015). Aus Perspektive des Serviceprozesses bearbeiten die Studierenden kooperativ mit den Partnern der gemeinnützigen Organisationen eine sozial relevante Problemstellung. Am Ende steht ein Serviceergebnis, was möglichst einen Beitrag zur Verbesserung der Lebensqualität in der Kommune leistet. Davon zu unterscheiden ist der Lernprozess, indem während der Problembearbeitung Konzepte und Methoden bzw. Inhalte des Studiums von den Studierenden erkundet und auf die Problemstellung angewendet werden. Hierbei ist das Lernergebnis vom Serviceergebnis zu differenzieren (vgl. dazu Tramm 2007, S. 119; Dilger 2011, S. 5). Das Lernergebnis zielt auf das generierte Wissen der Studierenden und welche persönlichen Einsichten diese hinsichtlich ihrer Werte und Einstellungen zu sozialen Herausforderungen erfahren haben.

\subsection{Wirksamkeit von Service Learning}

Die empirischen Studien zum Service Learning stammen hauptsächlich - vor allem im Bereich der wirtschaftswissenschaftlichen Bildung - aus dem US- 
amerikanischen und angelsächsischen Kontext. Dabei fällt auf, dass ein allgemeines Kompetenzmodell schwer zu identifizieren ist. Die Ergebnisse können quergelesen zu den Aspekten fachliche und methodische Kompetenz sowie Selbst- bzw. Humankompetenz zusammengefasst werden.

Hinsichtlich der fachlichen und methodischen Fähigkeiten zeigt sich in den empirischen Studien, dass durch Service Learning in der wirtschaftswissenschaftlichen Bildung v. a. Problemlösefähigkeiten, Selbstwirksamkeit, sozial-kommunikative Fähigkeiten und kritisches Denken gefördert werden können (vgl. u. a. Astin et al. 2000, Yorio \& Ye 2012). Prentice \& Robinson illustrieren aber über unterschiedliche Fächergruppen hinweg, dass die Studienleistungen sich von Service Learning-Teilnehmern nicht signifikant von Nicht-Service Learning-Teilnehmern unterscheiden (vgl. Prentice \& Robinson 2010). Reinders \& Wittek zeigen für den deutschsprachigen Kontext bei Studierenden der Psychologie, dass der subjektive Lernerfolg und die Handlungswirksamkeit beim Service Learning höher empfunden wird als bei traditionellen Verantstaltungsformen und Service Learning zur Veränderung des Selbstbildes und der persönlichen Einsichten beiträgt (vgl. Reinders \& Wittek 2009; auch Reinders 2010). Dieses Ergebnis zeigt sich auch in Studien aus dem US-amerikanischen Raum (vgl. Prentice \& Robinson 2010, Yorio \& Ye 2012). So konnte Burns (2011) zeigen, dass wenn Studierende ihr Serviceergebnis als nützlich für die Gesellschaft wahrnehmen, eine höhere Motivation haben, sich später in gemeinnützigen Organisationen zu engagieren.

Die Wirksamkeit von Service Learning wird häufig über die Lernergebnisse beschrieben. Der Zusammenhang zwischen didaktischer Gestaltung des Service Learning-Arrangements und die damit verbundene Wirkung wird demgegenüber weniger untersucht. So zeigen Yorio \& Ye (2012) in einer Meta-Studie auf, dass die curriculare Integration von Service Learning stärkere Effekte als ein extracurriculares Arrangement hat. Auch ist die wahrgenommene Kompetenzentwicklung höher, wenn die Studierenden ihr Serviceprojekt freiwillig wählen (vgl. Yorio \& Ye 2012). Auf der mikrodidaktischen Ebene illustrieren Prentice \& Robinson, dass das Aufzeigen der Verknüpfung zwischen Studieninhalten und Serviceprojekt (u. a. durch Dozenten) sowie ein fester Ansprechpartner für die Studierenden bei der gemeinnützigen Organisation relevant für den späteren Lernerfolg sind (vgl. Prentice \& Robinson 2010).

Insgesamt ist die Belastbarkeit der empirischen Ergebnisse unterschiedlich, da einerseits die untersuchten Service Learning-Arrangements konzeptionell verschieden ausgestaltet sind und in der Regel Service Learning-Kurse mit traditionellen Veranstaltungformen verglichen werden. Die Vieldimensionalität von Lernumgebungen wird selten aufgenommen. Andererseits kann aus methodischer Hinsicht festgehalten werden, dass die Messinstrumente heterogen sind und von geprüften Skalen (z. B. SELEB- 
Skala) über Eigenentwicklungen bis Fragebögen zur Veranstaltungsevaluation reichen (vgl. dazu Gerholz \& Losch 2015).

\subsection{Zwischenfazit}

Die Lernform Service Learning hat das Potenzial nicht nur fachliche und methodische Fähigkeiten im Studium zu stärken, sondern auch die Persönlichkeitsentwicklung der Studierenden durch die Verbindung zum gemeinnützigen Engagement zu fördern. Dies erfordert eine entsprechende didaktische Fundierung, indem eine kohärente Verknüpfung zwischen dem Serviceprozess und Lernprozess vorgenommen wird. Quergelesen zeigen die empirischen Studien trotz der Unterschiede der Belastbarkeit ihrer Befunde, dass die Potenziale von Service Learning erreicht werden können. Inwiefern die vorrangig aus dem US-amerikanischen Raum stammenden Befunde auch für die deutsche Universitätslandschaft zutreffend sind, stellt ein Forschungsdesiderat dar, da mit Service Learning nicht nur Lerneffekte verbunden werden, sondern auch der Beitrag zur Persönlichkeitsentwicklung näher zu untersuchen ist (vgl. Reeinders \& Wittek 2009).

Für die Tradition in der deutschsprachigen Universitätslandschaft stellt Service Learning eine neue Akzentuierung universitärer Bildung dar. Vor allem für den Kontext wirtschaftswissenschaftlicher Bildungsprozesse kann ein Forschungs- und Entwicklungsbedarf hinsichtlich Service Learning konstatiert werden. Auf curricularer Ebene sind Fragen der Möglichkeiten der Integration von Service Learning in Studien- und Bildungsgängen nachzugehen und auf mikrodidaktischer Ebene geht es um die Gestaltung von Service Learning-Formen und welche Wirkungen auf die Kompetenzentwicklung der Lernenden damit einhergehen. In einem ersten Schritt sollen dafür in der nachfolgenden Pilotstudie empirische Befunde zur Wahrnehmung der didaktischen Kohärenz von Service Learning bei Studierenden der Wirtschaftswissenschaften vorgestellt werden.

\section{Mixed Method-Studie zu Service Learning in der wirtschaftswissenschaftlichen Hochschulbildung}

\subsection{Kontext der Studie}

Die Fakultät für Wirtschaftswissenschaften an der Universität Paderborn bildet den Kontext der Studie. In den drei Bachelorstudiengängen (Wirtschaftswissenschaften, Wirtschaftsinformatik und International Business Studies) der Fakultät wurde im Sommersemester 2014 das Wahlpflichtmodul ,Service 
Learning in den Wirtschaftswissenschaften' angeboten. Der Aufbau des Moduls ist als Service Learning-Arrangement organisiert, in dem Studierende wirtschaftswissenschaftliche Problemstellungen bei gemeinnützigen Organisationen der Region bearbeiten (Beispiele sind die ,Entwicklung einer Fundraising-Toolbox für den Caritas-Verband' oder die ,Optimierung des Strategiekonzeptes für den Markplatz für Bürgerengagement'). Im Modul erarbeiten sich die Studierenden ein Wissen über wissenschaftliche Methoden und Verfahren der Wirtschafts- und Sozialwissenschaften. Basierend auf den Problemstellungen, wählen die Studierendengruppen entsprechende Methoden zu deren Bearbeitung aus, wenden diese auf die Problemstellung an, entwickeln auf Basis der Ergebnisse Lösungen für die gemeinnützigen Organisationen und reflektieren die Potenziale und Grenzen der eingesetzten Methoden (Beispiele sind $u$. a. die Durchführung einer SWOT-Analyse auf Basis einer Dokumentenanalyse, Bürgerbefragungen mit standardisierten Fragenbögen oder Experteninterviews mit kommunalen Verantwortungsträgern). Service Learning wurde somit als eine problembasierte Lernform umgesetzt. Über die Bearbeitung der Problemstellungen in den gemeinnützigen Organisationen sollen die Studierenden für soziale Herausforderungen in der Gesellschaft und der Relevanz von Verantwortungsübernahme sensibilisiert werden.

\subsection{Interesse der Studie und methodisches Design}

Das Interesse der Studie liegt in der Beschreibung der Motive und Engagementbereitschaft der Studierenden bezogen auf das beschriebene Service Learning-Modul und deren Erwartungen hinsichtlich ihrer Selbstwirksamkeit und subjektivem Lernerfolg. Es handelt sich um eine Ex-ante-Analyse, mit dem Ziel, die Konsistenz des didaktischen Designs zu beschreiben und Kriterien zur Evaluation von Service Learning-Arrangements zu entwickeln (vgl. Moorer 2009).

Methodisch wurde für die Ex-ante-Analyse eine Triangulation von quantitativen und qualitativen Datenformaten vorgenommen. Die Erhebung der quantitativen Datenformate wurde in einem ,Experimental-Kontrollgruppen'Design durchgeführt. Die Experimentalgruppe bildeten die Studierenden des Service Learning-Moduls und als Kontrollgruppe fungierte ein Methodenmodul der Bachelorstudiengänge mit einer traditionellen Seminarform. Die Studierenden beider Module bekamen jeweils nach der ersten Modulsitzung einen Fragebogen, in denen deren Selbstbild, Einstellung zum zivilgesellschaftlichen Engagement, erwartete Selbstwirksamkeit und erwarteten subjektiven Lernerfolg erfasst wurde; es wurden hier gängige Skalen aus dem Service Learning-Diskurs verwendet (vgl. u. a. Reinders 2010, Mabry 1998). Hinsichtlich der qualitativen Datenformate wurden mit jeweils zwei Studie- 
renden aus jeder der sechs Gruppen im Service Learning-Modul problemzentrierte Interviews nach der ersten Modulsitzung geführt (vgl. Witzel 2000), in denen ihre Einschätzungen und Erwartungen erhoben wurden. Die Interviews wurden transkribiert und nach der qualitativen Inhaltsanalyse der Strukturierung - nach Mayring ausgewertet (vgl. Mayring 2010).

\subsection{Ergebnisse}

\subsubsection{Wahrnehmung des Service Learning-Arrangements}

Tabelle 1 enthält die deskriptiven Kennwerte sowie die Reliabilitäten zu den erhobenenen Skalen; letztere sind zufriedenstellend (.78) bis gut (.84). Insgesamt zeigen die Studierenden im Service Learning-Modul höhere Ausprägungen als die Studierenden im Seminarmodul, wenngleich die Standardabweichungen dieses Bild relativieren. Eine größere Differenz zeigt sich bei dem erwarteten subjektiven Lernerfolg: Hier schätzen die Service LearningStudierenden sich fast eine Standardabweichung höher ein als die Studierenden im Seminarmodul.

Tab. 1: Beschreibung der Stichprobe (6 stufige Likert-Skala)

\begin{tabular}{|c|c|c|c|c|c|c|c|}
\hline \multirow[t]{2}{*}{ Skala } & \multirow[t]{2}{*}{ Items } & \multirow[t]{2}{*}{ Beispielitem } & \multirow[t]{2}{*}{$\alpha$} & \multicolumn{2}{|c|}{$\begin{array}{l}\text { SL-Modul } \\
(\mathrm{n}=39)\end{array}$} & \multicolumn{2}{|c|}{$\begin{array}{l}\text { Seminarmodul } \\
\qquad(\mathrm{n}=17)\end{array}$} \\
\hline & & & & MW & $\mathrm{SD}$ & MW & $\mathrm{SD}$ \\
\hline $\begin{array}{l}\text { Einstellung } \\
\text { zum zivil- } \\
\text { gesellschaftl. } \\
\text { Engagement }\end{array}$ & 5 & $\begin{array}{l}\text { Ungeachtet dessen ob } \\
\text { eine Person erfolgreich } \\
\text { war oder ist sollte sie } \\
\text { Anderen helfen. }\end{array}$ & .84 & 4.69 & .86 & 4.44 & .77 \\
\hline $\begin{array}{l}\text { Selbst- } \\
\text { wirksamkeit }\end{array}$ & 10 & $\begin{array}{l}\text { Es wird mir keine } \\
\text { Schwierigkeiten be- } \\
\text { reiten, meine Absichten } \\
\text { und Ziele im Modul zu } \\
\text { verwirklichen. }\end{array}$ & .87 & 4.30 & .69 & 4.18 & .67 \\
\hline Selbstbild & 5 & $\begin{array}{l}\text { Durch das Modul werde } \\
\text { ich mich selbst anders } \\
\text { als früher sehen. }\end{array}$ & .78 & 3.04 & 1.06 & 2.89 & .94 \\
\hline $\begin{array}{l}\text { Subjektiver } \\
\text { Lernerfolg }\end{array}$ & 7 & $\begin{array}{l}\text { Ich habe den Eindruck, } \\
\text { mein Wissen wird sich } \\
\text { langfristig erweitern. }\end{array}$ & .78 & 4.55 & .68 & 3.86 & .89 \\
\hline
\end{tabular}


In einer Varianzanalyse offenbart sich ein signifikanter, moderater Effekt beim erwarteten Lernerfolg $\left(F_{(1,53)}=9.554, \mathrm{p}<.01, \eta^{2}=.160\right)$; weitere signifikante Effekte ergeben sich zwischen den Gruppen nicht. Die Service Learning-Studierenden erhoffen sich somit einen höheren Lernerfolg als die Studierenden im Seminarmodul.

\subsubsection{Gründe für die Modulwahl Service Learning}

Die Studierenden im Service Learning-Modul wurden im Fragebogen u. a. nach den Gründen für die Wahl des Moduls gefragt. Hierbei ging es darum, inwiefern der Anwendungsbezug (,Es war mir wichtig, einen praktischen Anwendungsbezug im Modul zu haben.') oder der soziale bzw. gemeinnützige Bezug (,Ich finde es wichtig, die Möglichkeit zu haben, gemeinnützigen Organisationen helfen zu können.') leitend bei der Modulwahl war. Eine Korrelationsanalyse zeigt ein signifikanten mittleren Zusammenhang zwischen Anwendungsbezug und erwarteten subjektiven Lernerfolg $(\mathrm{r}=0,481, \mathrm{p}$ $<0,05)$, während kein signifikanter Zusammenhang beim sozialen Bezug vorliegt $(r=0,324)$. Dieser Zusammenhang spiegelt sich auch in der Auswertung der qualitativen Datenformate wider (vgl. Tabelle 2). Die Kodierungen der Gründe für die Wahl des Service Learning-Moduls können vier Kategorien zugeordnet werden. Über $50 \%$ der Kodierungen liegen in der Kategorie ,Anwendungsbezug', während $6 \%$ im Bereich ,sozialer Bezug' einzuordnen sind. Als weitere Gründe wurden von den Studierenden die Form des ,Assessments' sowie ,pragmatische Gründe' angeführt.

Tab. 2: Gründe für die Wahl des Moduls Service Learning (SL)

\begin{tabular}{lccl}
\hline $\begin{array}{l}\text { Gründe Modul- } \\
\text { wahl SL }\end{array}$ & absolut & relativ & Ankerbeispiele \\
\hline $\begin{array}{l}\text { Anwendungs- } \\
\text { bezug }\end{array}$ & 15 & 0,52 & $\begin{array}{l}\text { "Also bei mir ist es definitiv der Praxis- } \\
\text { bezug“; ,Der Hauptpunkt ist schon der } \\
\text { Praxischarakter gewesen.“ }\end{array}$ \\
\hline Sozialer Bezug & 2 & 0,06 & $\begin{array}{l}\text {,ja hat halt diesen sozialen Aspekt und mit } \\
\text { gemeinnützigen Organisationen“; , dass man } \\
\text { auch einfach mal mit Hilfsorganisationen } \\
\text { arbeitet" }\end{array}$ \\
\hline Assessment & 5 & 0,17 & $\begin{array}{l}\text { "wir machen (..) die Ausarbeitung und das ist } \\
\text { dann auch nicht mehr so viel Arbeit."; ,schon } \\
\text { ganz nett zum Schluss eine Klausur weniger } \\
\text { zu haben“ }\end{array}$ \\
\hline Pragmatismus & 7 & 0,24 & $\begin{array}{l}\text { "dass ich kein Statistik machen muss.“; ,Weil } \\
\text { ich in ein anderes Modul nicht reingekommen } \\
\text { bin“ }\end{array}$ \\
\hline
\end{tabular}


Insgesamt zeigt sich somit das Bild, dass der Anwendungsbezug im Service Learning-Modul stärker leitend bei der Modulwahl ist als die Tatsache, dass die Problemstellungen einen gemeinnützigen und caritativen Bezug haben.

\subsubsection{Wahrgenommenes didaktisches Design in den Serviceprojekten}

Im Service Learning-Modul bearbeiten die Studierenden jeweils in 6-8er Personengruppen sechs unterschiedliche Serviceprojekte. Bei der didaktischen Konzeption wurde darauf geachtet, dass der Anforderungsgrad in den jeweiligen Problemstellungen strukturell ähnlich ist. Hierbei ergibt sich die Frage, wie die einzelnen Studierendengruppen ihre Serviceprojekte hinsichtlich ihrer eigenen Wirksamkeit und den erwarteten subjektiven Lernerfolg einschätzen. In Abbildung 1 sind die Mittelwerte hinsichtlich erwarteter Selbstwirksamkeit und erwarteten Lernerfolg abgebildet.

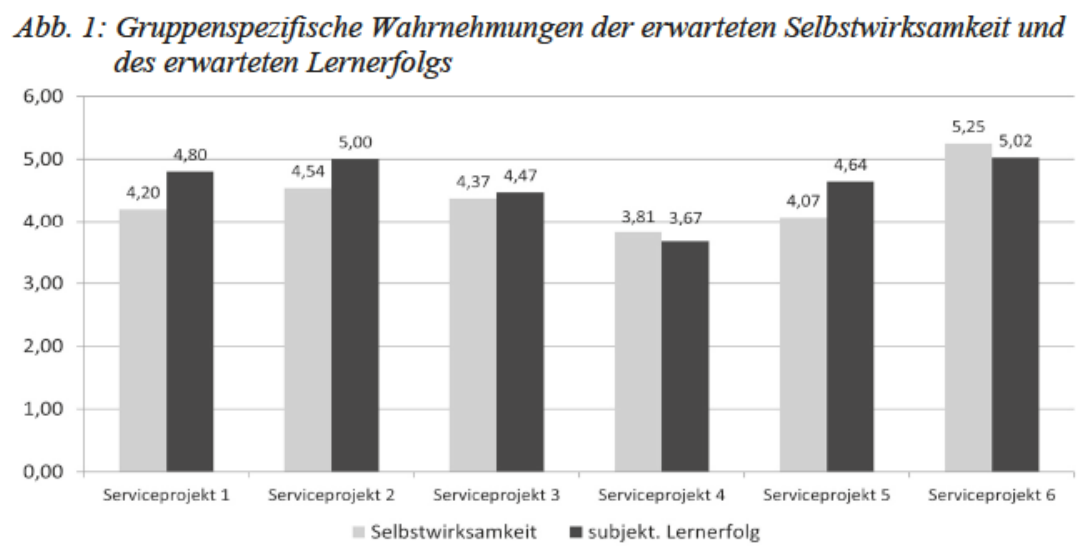

Quelle: eigene Darstellung

Deskriptiv weisen die Gruppen Unterschiede auf. In einer Varianzanalyse zeigt sich, dass die Unterschiede sowohl beim erwarteten Lernerfolg ( $F$ $\left.(5,36)=5,836, \mathrm{p}<.001, \eta^{2}=.493\right)$ als auch bei der erwarteten Selbstwirksamkeit $\left(F(5,36)=4,567, \mathrm{p}<.01, \eta^{2}=.432\right)$ signifikant sind und mit moderaten Effekten einhergehen. Die Gruppenzugehörigkeit oder das zu bearbeitende Serviceprojekt hat somit einen Einfluss auf den zugeschriebenen Lernerfolg und die empfundene Wirksamkeit des eigenen Handelns. 
Die Ergebnisse der Interviewauswertung erhellen die Unterschiede zwischen den Gruppen. ${ }^{1}$ Die Studierenden wurden u. a. in den Interviews gefragt, welche Fähigkeiten sie durch das Service Learning-Modul weiterentwickeln werden (vgl. Tabelle 3). Insgesamt erwarten die Studierenden einen Zuwachs bei den fachlich-methodischen Fähigkeiten, gefolgt von den sozialkommunikativen und personalen Fähigkeiten, wenngleich die Nennungen nahezu gleich verteilt sind. Es zeigen sich hier Gemeinsamkeiten mit den Ergebnissen der empirischen Studien aus dem US-amerikanischen Raum (vgl. Abschnitt 2.2). Neben den Problemlöse- und kommunikativen Fähigkeiten erwarten die Studierenden auch eine Veränderung ihrer personalen Fähigkeiten, was sich u. a. in der Übernahme von Verantwortung oder Einblick in caritative Bereiche äußert. Insgesamt finden sich in den Interviewdaten keine Hinweise, dass der Fähigkeitszuwachs unterschiedlich in den einzelnen Gruppen gesehen wird, was aufgrund der signifikanten Unterschiede im erwarteten subjektiven Lernerfolg zu erwarten wäre.

\section{Tab. 3: Erwarteter Fähigkeitszuwachs von Service Learning-Studierenden}

\begin{tabular}{|c|c|c|c|}
\hline $\begin{array}{l}\text { Erwartete } \\
\text { Fähigkeiten }\end{array}$ & absolut & relativ & Ankerbeispiele \\
\hline $\begin{array}{l}\text { Fachlich- } \\
\text { methodische } \\
\text { Fähigkeiten }\end{array}$ & 11 & 0,42 & $\begin{array}{l}\text { „Überhaupt methodisches Fachwissen } \\
\text { oder das auch anzuwenden“; ,Ich denke } \\
\text { die Methodik auf jeden Fall.“ }\end{array}$ \\
\hline $\begin{array}{l}\text { Sozial- } \\
\text { kommunikative } \\
\text { Fähigkeiten }\end{array}$ & 8 & 0,31 & $\begin{array}{l}\text { „Gesprächsfiuhrung schon ein wenig } \\
\text { weiterentwickelt"; ,,soziale Bereich durch } \\
\text { die Kooperationspartner, dass man da in } \\
\text { Kontakt tritt" }\end{array}$ \\
\hline $\begin{array}{l}\text { Personale } \\
\text { Fähigkeiten }\end{array}$ & 7 & 0,27 & $\begin{array}{l}\text { „für solche Sachen Verantwortung } \\
\text { tragen“; , ,also Präsentationen, das ist } \\
\text { nicht mein Ding (...) das jetzt mal zu } \\
\text { machen, ob es funktioniert" }\end{array}$ \\
\hline
\end{tabular}

Weiterhin wurden die Studierenden gefragt, welche Handlungsherausforderungen sie im Service Learning-Modul sehen. Die Kategorie ,Organisation und Koordination', worunter u. a. die Gruppenarbeit, die Kooperation mit den gemeinnützigen Organisationen und den Modulverantwortlichen subsumiert werden können, wird mehrheitlich positiv gesehen: 28 positive Nennungen (z. B. „wir haben relativ wenige Probleme mit unserem Praxis-

1 Ziel der Kontrastierung der quantiativen und qualitativen Daten ist die Herausarbeitung der individuellen und gruppenspezifischen Phänomene, um u. a. ein Verständnis für die Ergebnisse der quantiativen Daten zu generieren. Dies ist nicht im Sinne der Aufdeckung von Kausalitäten zu verstehen, da hierfür die paradigmatischen Orientierungen in den Blickpunkt gerückt werden müssen; hier liegen die Grenzen in Mixed Method-Ansätzen. 
partner. “, „, Gruppe läuft wunderbar, dass ist alles harmonisch”) versus 9 negative Nennungen (z. B. ,wenn ich das alleine machen würden, würde ich schneller vorankommen"). Stärker werden Herausforderungen in der Bearbeitung des Serviceprojektes wahrgenommen, bei denen von den 24 Hinweisen im Datenmaterial 22 als herausfordernd eingestuft werden können. Hierbei beziehen sich die Nennungen sowohl auf den Serviceprozess (,dass die vom Marktplatz sehr viel verlangen und wir das in der kurzen Zeit gar nicht erfüllen können ") als auch auf die Verknüpfung zu den fachlichen Inhalten (z. B. ,wir haben tierische Probleme die Problemstellung einzugrenzen"; „,das Fachliche, was machen wir wirklich (.) da wird das Problem liegen."). Allerdings zeigt sich in den Nennungen der einzelnen Gruppen keine Tendenz, die ein besseres Verständnis für die Unterschiede in der erwarteten Selbstwirksamkeit hervorbringt.

\section{Zusammenführung der Ergebnisse und Ausblick}

Zusammenführend zeigen die Ergebnisse der Ex-ante-Analyse, dass der Veranstaltungsform Service Learning ein höherer Lernerfolg seitens der Studierenden zugeschrieben wird als die Veranstaltungsform Seminar. Die Gründe dafür liegen weniger im Bezug zu caritativen Kontexten, sondern im stärkeren Anwendungsbezug in Form der Bearbeitung realer Problemstellungen. Hinsichtlich der didaktischen Konsistenz zeigen die Ergebnisse, dass die Gruppenzugehörigkeit und die einzelnen Serviceprojekte mit der erwarteten Wirksamkeit des eigenen Handelns und den zugeschriebenen Lernerfolg korrespondieren. Die Ergebnisse der Interviewauswertung generieren ein erstes Verständnis für die Unterschiede, wenngleich eine klare Tendenz sich nicht abzeichnet. So gibt es Hinweise, dass die Studierenden sowohl Herausforderungen im Serviceprozess als auch in der Gestaltung des Lernprozesses sehen, was die Relevanz von didaktischen Unterstützungsmaßnahmen sowohl im Serviceprozess (z. B. Organisation der Austauschprozesse zwischen Studierenden, Lehrenden und gemeinnützigen Organisationen) als auch in der Begleitung der Lernprozesse (z. B. Verknüpfung zwischen Studieninhalten und Serviceprojekt, Beratungsleistungen) unterstreicht.

Die Befunde zeigen für die zukünftige Erforschung von Service Learning-Arrangements, dass die Zusammenhänge zwischen Problemstellung im Serviceprojekt, die Einschätzungen der Studierenden und die didaktische Aufbereitung der Studieninhalte näher in den Blick zu nehmen sind. Es geht um die didaktische Kohärenz eines Service Learning-Arrangements. Darüber hinaus weisen die Befunde darauf hin, dass Service Learning das Potenzial hat, einen subjektiv höheren Lernerfolg zu generieren, gleichzeitig die Motive der Studierenden stärker im Anwendungsbezug als im gemeinnützigen 
Bezug liegen. Eine Erklärung für dieses Phänomen kann die im Vergleich zum US-amerikanischen Raum geringere Ausprägung einer GemeinwohlOrientierung in der deutschen Universitätslandschaft sein. Hierbei ist zu berücksichtigen, dass über Service Learning genau die Verbindung zwischen Gemeinwohl- und Anwendungsbezug hergestellt werden kann. Ein Ziel wäre es somit, Studierende im deutschsprachigen Raum stärker für die Relevanz sozialer Probleme in der Gesellschaft zu sensibilisieren, um damit auch zu vermeiden, dass Service Learning ,nur' als Lernen mit realen Problemstellungen ohne den konstituierenden Bezug zu gemeinnützigen Handlungsfeldern verbunden wird.

\section{Literatur}

Astin, A. W., Vogelgesang, L. J., Ikeda, E. K., \& Yee, J. A. (2000). How servicelearning affects students. University of California, LA: Higher Education Research Institute.

Backhaus-Maul, H. \& Roth, C. (2013): Service Learning an Hochschulen in Deutschland. Ein erster empirischer Beitrag zur Vermessung eines jungen Phänomens. Wiesbaden: Springer VS.

Bringle, R. G. \& Clayton, P. H. (2012). Civic Education through Service Learning: What, How, and Why?. In L. McIlrath, A. Lyons \& R. Munck (Hrsg.), Higher Education and Civic Engagement. Comparative Perspectives (S. 101-123). New York.

Burns, D. J. (2011). Motivations to volunteer and benefits from Service Learning: An exploration of marketing students. Journal for Advancement of Marketing Education, 18, 10-23.

Dewey, J. (1998). Experience and education. West Lafayette, Indiana: Kappa Delta (Original published 1938).

Dewey, J. (1966). Democarcy and education. New York: Free Press (Original published 1916).

Dilger, B. (2011): Die Probleme mit den Problemen: Oder Missverständnisse bei der Konstruktion von Lernsituationen. Berufs- und Wirtschaftspädagogik - online, Ausgabe 20, 1-21. Online: http://www.bwpat.de/ausgabe20/dilger_bwpat20.pdf (19.10.2014).

Gerholz, K.-H. \& Losch, S. (2015): Can service learning foster a social responsibility among students? - A didactical analysis and empirical case-study in business education at a German university. In: L. O'Riordan, S. Heinemann \& P. Zmuda, (Hrsg.), Corporate Social Responsibility: Locating the Missing Link, (S. 599622). Wiesbaden.

Godfrey, P. C., Illes, L. M. \& Berry, G. R. (2005). Creating Breadth in Business Education trough Service-Learning. Academy of Management Learning \& Education, 4(3), 309-323. 
Mabry, J. B. (1998). Pedagogical Variations In Service-Learning And Student Outcomes: How Time, Contact, And Reflection Matter. Michigan Journal of Community Service Learning, 5, 32-47.

Mayring, P. (2010): Qualitative Inhaltsanalyse. Grundlagen und Techniken (11. Aufl.). Weinheim etc.

Moorer, C. (2009). Service Learning and Business Education: Distinctions between undergraduate and graduate business students. American Journal of Business Education, 2(3), 63-72.

Muller, S. (1999). Deutsche und Amerikanische Universitäten im Zeitalter der Kalkulation. In M. G. Ash (Hrsg.), Mythos Humboldt: Vergangenheit und Zukunft der deutschen Universitäten (S. 195-199). Wien, Köln, Weimar: Böhlau.

Prentice, M. \& Robinson, G. (2010). Improving Student Learning Outcomes with Service Learning. American Association of Community Colleges, Online: http:// www.aacc.nche.edu/Resources/aaccprograms/horizons/Documents/slorb_jan201 0.pdf (27.01.2014).

Reinders (2010). Lernprozesse durch Service Learning an Universitäten. Zeitschrift für Pädagogik, 56(4), 531-547.

Reinders, H. \& Wittek, R. (2009). Persönlichkeitsentwicklung durch Service Learning an Universitäten. In K. Altenschmidt, J. Miller \& W. Stark (Hrsg.), Raus aus dem Elfenbeintrum? (S. 128-143), Weinheim, Basel: Beltz.

Tramm, T. (2007). Im Lernfeld selbständig Probleme lösen? Oder: Von der Unmöglichkeit, sich am eigenen Schopf aus dem Sumpf zu ziehen. In: F.-W. Horst, J. Schmitter \& J. Tölle (Hrsg.), Wie MOSEL Probleme löst. Band 1, Lernarrangements wirksam gestalten. Paderborn, 104-138.

Yorio, P. L. \& Ye, F. (2012). A Meta-Analysis on the Effects of Service-Learning on the Social, Personal, and Cognitive Outcames of Learning. Academy of Management Learning \& Education, 11(1), 9-27. 